\title{
Online Farsi Handwritten Character Recognition Using Hidden Markov Model
}

\author{
Vahid Ghods*, Mohammad Karim Sohrabi \\ Department of Electrical and Computer Engineering, Semnan Branch, Islamic Azad University, Semnan, Iran. \\ *Corresponding author. Tel:+98 23 33654040; email: v.ghods@semnaniau.ac.ir \\ Manuscript submitted April 5, 2015; accepted July 10, 2015. \\ doi: $10.17706 /$ jcp.11.2.169-175
}

\begin{abstract}
In this paper, a method for recognizing online Farsi characters that are written separately has been introduced. Regarding to the shape and the structure of the main body, Farsi letters have been divided into 18 groups. First, hidden Markov model (HMM) technique has been exploited to recognize the main body. In the next step, the final recognition in each group is performed according to delayed strokes (dots and small signs) and their hidden Markov models. The proposed method has been tested on TMU dataset and the recognition accuracy of $95.9 \%$ and $94.2 \%$ has been obtained for the recognition of the group and the character, respectively.
\end{abstract}

Key words: Character recognition, Farsi, Persian, online handwriting, HMM.

\section{Introduction}

According to the received information, handwriting recognition divided into offline and online [1]. Offline recognition of Farsi handwriting has much research; however, little research has been done in recognition of online handwriting [2]-[5]. In online recognition, coordinates of the pen movement, number of the pen movements and pen pressure are available. Online recognition is met special attention because of more convenience writing than typing, unable to type in some situations, lack of a full keyboard on small computers, and hard typing in some languages due to their large number of letters. Usually, hidden Markov model , dynamic programming and neural networks are employed for the recognition of Farsi characters and numbers [6], [7]. In [8], online Farsi recognition has been performed with a fuzzy approach. Grouping and recognition of Farsi characters are presented by [9] using the structural characteristics. In the paper, 24 new online features are presented.

In this paper, the first step to recognize the Farsi letters is grouping using hidden Markov models of their main bodies. Next, using the number and the position of the delayed strokes and their models (if needed) result to the final recognition. We implemented the proposed method on TMU database [10].

In the second section, the letters are grouped according to the main body and the final recognition is performed by helping the delayed strokes. In the third section, experimental results are expressed on more than 4000 online Farsi characters. The fourth section concludes the paper.

\section{Character Grouping and Recognition}

Based on a variety of the Farsi letter writing, we formed 18 groups of the main body. The members of each group have the same shape and characteristics. Members of these groups are shown in Table 1. At this stage, 
according to the same main body and having an adequate sample from each group, continuous hidden Markov model was used. The number of states and mixtures were considered 16 and 4, respectively.

Table 1. Determined Groups for the Characters

\begin{tabular}{|c|c|c|c|c|c|c|c|c|c|c|}
\hline Group 1 & \multicolumn{2}{|c|}{$\begin{array}{c}1 \\
\text { Alef }\end{array}$} & \multicolumn{2}{|c|}{$\begin{array}{c}\text { i } \\
\text { Alef with } \\
\text { hat }\end{array}$} & \multirow{2}{*}{$\begin{array}{l}\text { Group } 7 \\
\text { Group } 8\end{array}$} & \multirow{2}{*}{$\begin{array}{c}\text { ضaad } \\
\text { ظ } \\
\text { Zaa }\end{array}$} & \multirow{2}{*}{$\begin{array}{c}\text { ص } \\
\text { Saad } \\
b \\
\text { Taa }\end{array}$} & \multirow{2}{*}{$\begin{array}{l}\text { Group } 13 \\
\text { Group } 14\end{array}$} & \multicolumn{2}{|c|}{$\begin{array}{c}r \\
\text { Mim }\end{array}$} \\
\hline Group 2 & $\begin{array}{l}\dot{\leftrightarrow} \\
\mathrm{Se}\end{array}$ & $\begin{array}{c}ت \\
\mathrm{Te}\end{array}$ & $\begin{array}{l}\ddot{P} \\
\mathrm{Pe}\end{array}$ & $\begin{array}{c} \\
\mathrm{Be}\end{array}$ & & & & & & \\
\hline Group 3 & $\begin{array}{c}\dot{\tau} \\
\text { Khe }\end{array}$ & $\begin{array}{c}\tau \\
\mathrm{He} \\
\text { jimi }\end{array}$ & $\begin{array}{c}\text { 巨 } \\
\text { Che }\end{array}$ & $\begin{array}{c}\text { ج } \\
\text { Jim }\end{array}$ & Group 9 & $\begin{array}{c}\dot{\varepsilon} \\
\text { Ghein }\end{array}$ & $\begin{array}{c}\varepsilon \\
\text { Ein }\end{array}$ & Group 15 & & \\
\hline Group 4 & $\mathrm{Za}$ & & $\mathrm{Da}$ & & Group 10 & & & Group 16 & & \\
\hline Group 5 & $\begin{array}{c}j \\
\text { Zhe }\end{array}$ & & & $\begin{array}{l}J \\
\operatorname{Re}\end{array}$ & Group 11 & & & Group 17 & & \\
\hline Group 6 & $\mathrm{Sh}$ & & $\mathrm{Si}$ & & Group 12 & & & Group 18 & $\begin{array}{c}\xi \\
\text { Gaaf }\end{array}$ & $\begin{array}{c}\varsigma \\
\text { Kaaf }\end{array}$ \\
\hline
\end{tabular}

Online features to extract the features of the main body are as follows [11]:

-Coordinates of normalized ' $x$ ';

-Coordinates of normalized ' $y$ ';

$-\theta$, the angle between the straight line between two sequential sampled points and the $\mathrm{x}$-axis;

$-\Delta \theta$, difference of current angle $\theta$ with the previous one;

-Sin $(\theta)$, writing direction;

-Cos $(\theta)$, writing direction;

-Sin $(\Delta \theta)$, curvature;

- $\operatorname{Cos}(\Delta \theta)$, curvature;

$-V x$, speed along the $x$-axis;

-Vy, speed along the y-axis;

-Vicinity Aspect;

-Vicinity Slope;

-Euclidean distance of each sample to the line between the first and the last samples.

Single-member groups (groups 10 to 17) were recognized completely in this stage. Double-members groups (groups 1, 4, 6, 7, 8, 9, 18) with respect to the number of pen movements were recognized finally. For example, if the input character is classified in group 6, if there is no delayed stroke (number of the pen movement is 1), the letter is "س" ("Sin") and if there is a delayed stroke (number of the pen movement is more than 1), the letter "ش" ("Shin") is the ultimate recognition answer. The proposed algorithm is shown in Fig. 1.

If the input character is classified in groups 2, 3 and 5, the hidden Markov model of the delayed strokes (dots and signs) were exploited to complete the final recognition. At this stage, the continuous HMM is also used. Due to the less complexity of the delayed stokes data, the number of states and mixtures were considered 4 and 2, respectively. Online features to extract the characteristics of the delayed strokes are as follows [11]: 
-Coordinates of normalized ' $x$ ';

-Coordinates of normalized ' $y$ ';

$-\theta$, the angle between the straight line between two sequential sampled points and the $\mathrm{x}$-axis;

$-\Delta \theta$, difference of current angle $\theta$ with the previous one;

-Sin $(\theta)$, writing direction;

- $\operatorname{Cos}(\theta)$, writing direction;

-Sin $(\Delta \theta)$, curvature;

- $\operatorname{Cos}(\Delta \theta)$, curvature;

$-V x$, speed along the $x$-axis;

$-V y$, speed along the $y$-axis;

-Vicinity Aspect;

-Vicinity Slope;

-Normalized area of the delayed stroke;

-Ratio of the delayed stroke area to its main body area.

In final step, with respect to the number of the delayed strokes and their position (top, center and down), the input character was recognized by assisting the HMM of the delayed strokes for the groups of 2,3 and 5 . In group5, if the number of stroke is one, the recognized character is " $ر "$ ("Re"). If the number of strokes is more, the character is recognized based on its delayed stroke model Meanwhile, all characters are recognized regarding their delayed stroke models and positions in group 2. In group 3, if the number of stroke is one, the recognized character is "ح" ("He jimi"), otherwise the delayed stroke model determines the final recognition.

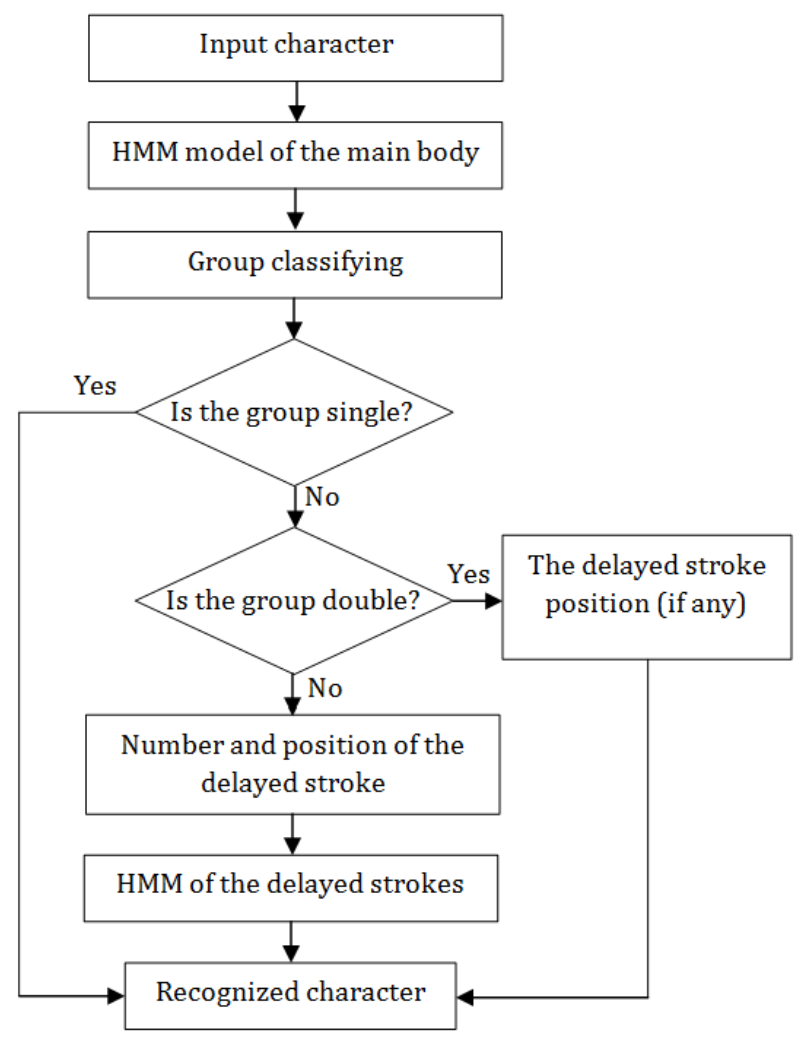

Fig. 1. The proposed method flowchart.

\section{Experimental Result}

If In this study, the TMU dataset was used with 4000 online Farsi handwritten letters that were written by 
120 writers [10]. In this collection, 117 samples of each letter are available. WACOM GRAPHIRE pen and pad were used to collect data. 60 samples of each letter (51\%) were considered as a training set and the other samples (57 samples) as test set.

The results of the implementation of the proposed method for determining the samples belong to their appropriate groups, according to the groups of Table 1, and the final character recognition are shown in Tables 2 and 3, respectively. As it can be see, the group recognition accuracy of $95.9 \%$ and final recognition accuracy of $94.2 \%$ are achieved.

Table 2. Confusion Matrix of Group Classification (Total Accuracy $=95.9 \%$ (for Grouping))

\begin{tabular}{|c|c|c|c|c|c|c|c|c|c|c|c|c|c|c|c|c|c|c|}
\hline $\begin{array}{c}\text { Qut } \\
\text { In }\end{array}$ & 1 & 2 & 3 & 4 & 5 & 6 & 7 & 8 & 9 & 10 & 11 & 12 & 13 & 14 & 15 & 16 & 17 & 18 \\
\hline 1 & 111 & 0 & 0 & 0 & 2 & 0 & 0 & 0 & 0 & 0 & 0 & 0 & 1 & 0 & 0 & 0 & 0 & 0 \\
\hline 2 & 0 & 217 & 0 & 1 & 0 & 1 & 0 & 0 & 0 & 2 & 2 & 0 & 0 & 3 & 0 & 0 & 0 & 2 \\
\hline 3 & 0 & 0 & 221 & 0 & 0 & 0 & 0 & 0 & 5 & 0 & 0 & 0 & 1 & 0 & 0 & 1 & 0 & 0 \\
\hline 4 & 0 & 1 & 0 & 112 & 1 & 0 & 0 & 0 & 0 & 0 & 0 & 0 & 0 & 0 & 0 & 0 & 0 & 0 \\
\hline 5 & 1 & 4 & 0 & 3 & 163 & 0 & 0 & 0 & 0 & 0 & 0 & 0 & 0 & 0 & 0 & 0 & 0 & 0 \\
\hline 6 & 0 & 1 & 0 & 0 & 0 & 110 & 0 & 0 & 0 & 0 & 0 & 0 & 0 & 0 & 0 & 0 & 3 & 0 \\
\hline 7 & 0 & 0 & 0 & 0 & 1 & 1 & 111 & 0 & 0 & 1 & 0 & 0 & 0 & 0 & 0 & 0 & 0 & 0 \\
\hline 8 & 0 & 0 & 0 & 0 & 0 & 0 & 0 & 107 & 0 & 3 & 0 & 0 & 0 & 0 & 0 & 4 & 0 & 0 \\
\hline 9 & 0 & 0 & 3 & 0 & 0 & 0 & 0 & 0 & 111 & 0 & 0 & 0 & 0 & 0 & 0 & 0 & 0 & 0 \\
\hline 10 & 0 & 2 & 0 & 0 & 0 & 0 & 1 & 0 & 0 & 53 & 1 & 0 & 0 & 0 & 0 & 0 & 0 & 0 \\
\hline 11 & 0 & 0 & 0 & 0 & 0 & 1 & 2 & 0 & 0 & 1 & 53 & 0 & 0 & 0 & 0 & 0 & 0 & 0 \\
\hline 12 & 0 & 1 & 0 & 0 & 0 & 0 & 0 & 0 & 0 & 0 & 0 & 55 & 0 & 0 & 0 & 1 & 0 & 0 \\
\hline 13 & 0 & 0 & 0 & 0 & 0 & 0 & 0 & 0 & 0 & 0 & 0 & 0 & 56 & 0 & 1 & 0 & 0 & 0 \\
\hline 14 & 0 & 1 & 0 & 0 & 0 & 0 & 0 & 0 & 0 & 0 & 3 & 1 & 0 & 51 & 0 & 0 & 1 & 0 \\
\hline 15 & 0 & 0 & 0 & 0 & 0 & 0 & 0 & 0 & 0 & 1 & 0 & 0 & 0 & 0 & 56 & 0 & 0 & 0 \\
\hline 16 & 0 & 0 & 0 & 1 & 0 & 0 & 0 & 3 & 0 & 0 & 0 & 1 & 0 & 0 & 0 & 52 & 0 & 0 \\
\hline 17 & 0 & 0 & 0 & 0 & 1 & 1 & 1 & 1 & 0 & 0 & 0 & 0 & 0 & 0 & 0 & 0 & 53 & 0 \\
\hline 18 & 0 & 1 & 0 & 0 & 0 & 0 & 0 & 0 & 0 & 1 & 0 & 0 & 0 & 0 & 0 & 0 & 0 & 112 \\
\hline
\end{tabular}

Table 3. Confusion Matrix of Final Character Recognition (Total Accuracy $=94.2 \%$ (for Recognition))

(The names of Farsi characters were introduced in Table 1.)

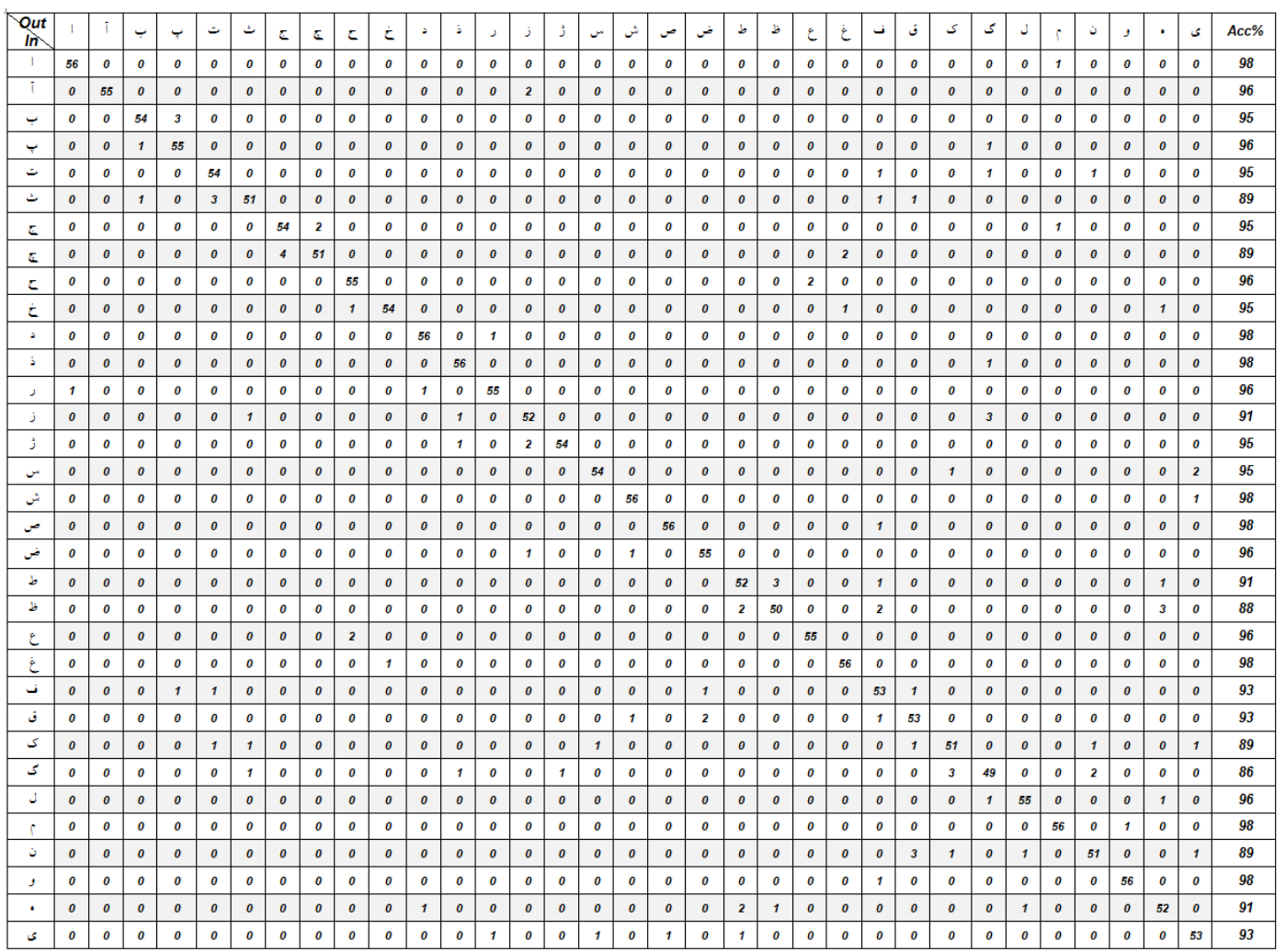

Examples of errors to recognize the appropriate group of the main body is analyzed. In Fig. 2, examples of "ى" ("Ye") are shown that belong to the group 17, however have not been recognized properly. In this work, these unusual manuscripts were ignored. 
Problem in recognizing the group 8 is a variety of writing style of these two letters by different people. In the dataset, four different styles of writing have seen that given in Fig. 3. In this study, the form of Fig. 3(a) was trained.

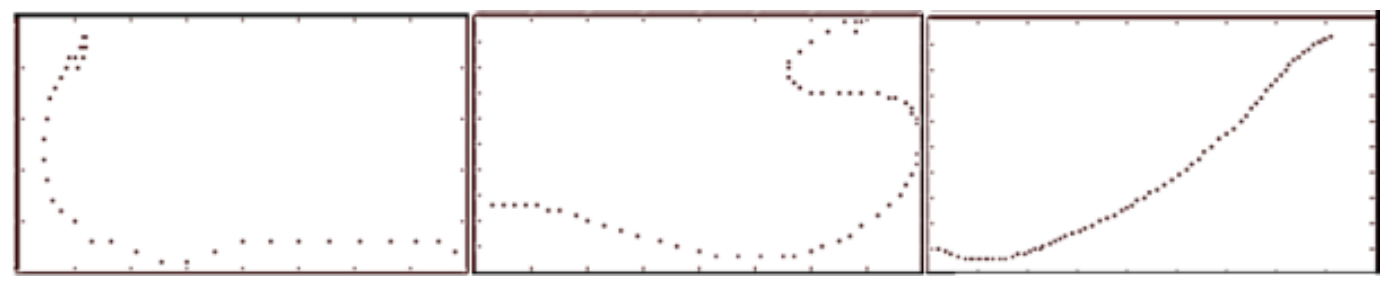

Fig. 2. Samples of "ى" ("Ye") letter that wrongly classified [4].

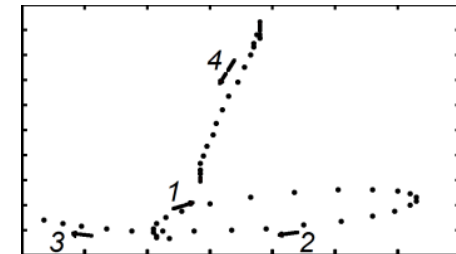

(a)

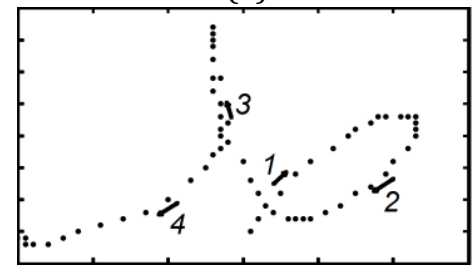

(c)

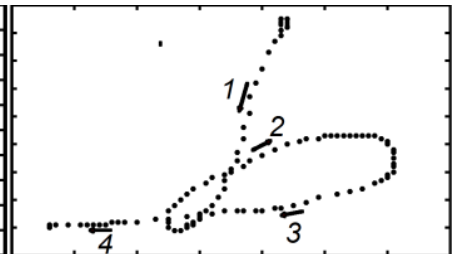

(b)

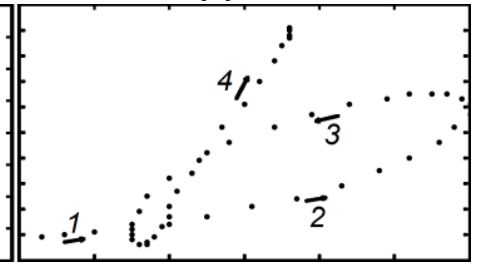

(d)

Fig. 3. Diversity of writng styles in "ط" ("Taa") letter [4].

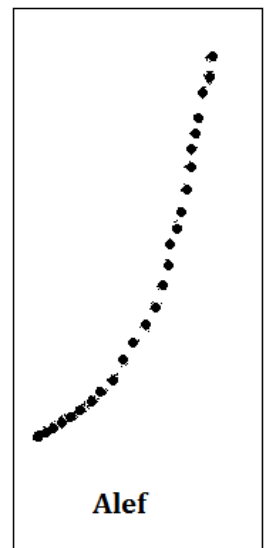

Fig. 4. This "I" ("Alef") is wrongly recognized in group 4.

Some members of the group 1 are recognized incorrectly in group 4 due to a skewed writing of "I" ("Alef") by the author (Fig. 4). An important cause of error in groups 3 and 9 recognition is more similarity of members of these groups that increases mistakes in both groups recognition (Fig. 5). Several members of group 5 have been classified incorrectly in groups 2 and 4 . In group 8, because of taken into consideration first pen stroke (main body), some members of these groups were mistakenly recognized in another group (group 8 in 16 or vice versa, see Fig. 6).

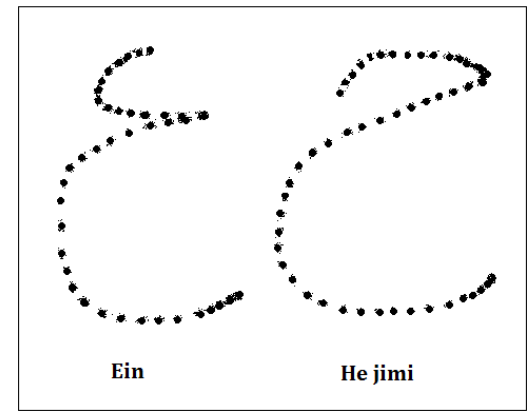

Fig. 5. The similarity of the main body shapes in groups 3 and 9.

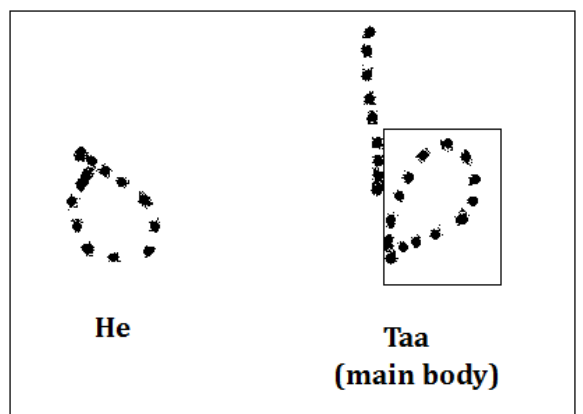

Fig. 6. Some main bodies of group 8 wrongly recognized in group 16 and vice versa.

Other types of errors occurred at the recognition of the delayed stroke [12]. In Fig. 7(a), single-dot of letter "ب" ("Be") was recognized wrongly with triple-dots because writer was rotated the pen. In Fig. 7(b), due to the large curvature in writing, double-dots has been detected triple-dots and made an error. In Fig. 7(c), the pen has slipped while single-dot writing and wrongly recognized triple-dots. In comparison the proposed method with previous methods, in spite of using the position of the delayed strokes (top, center and down), 
some delayed strokes HMMs were employed to enhance the recognition.

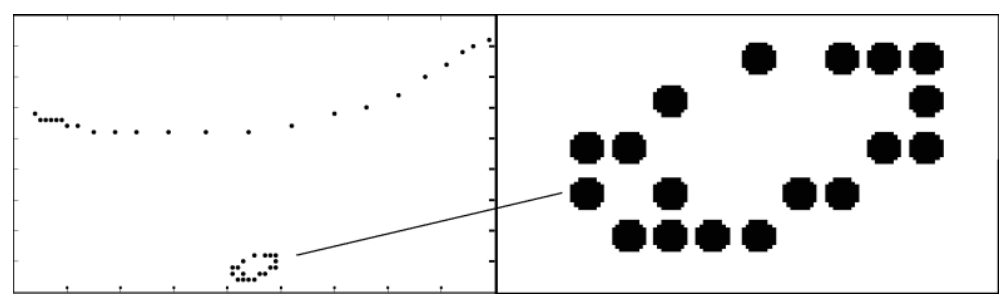

(a) Single-dot in "ب" ("Be") letter

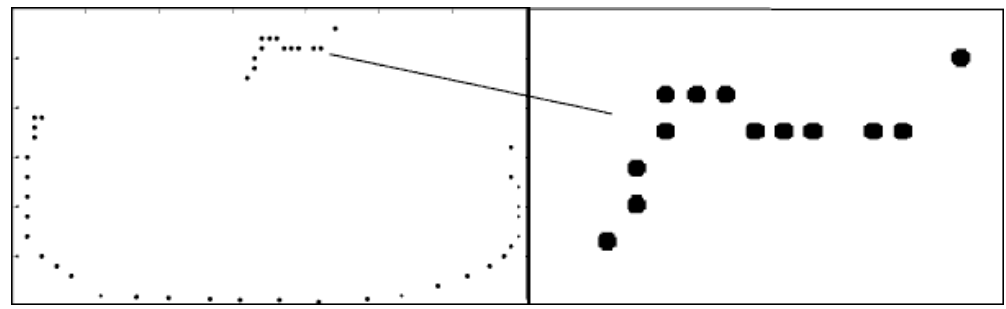

(b) Double-dots in "ت" ("Te") letter

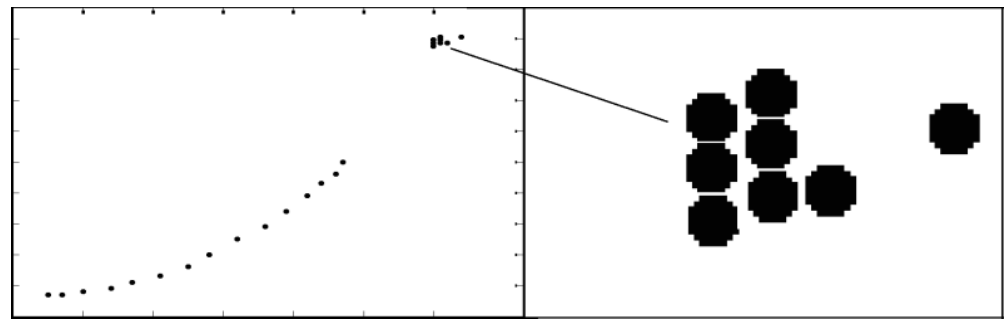

(c) Single-dot in "j" ("Ze") letter

Fig. 7. Samples of wrong recognition in the dots.

\section{Conclusion}

This paper presents an efficient technique for grouping and recognition of Farsi characters. The algorithm described the first movement of the pen (the main body of the letter) was used for grouping and the next movements (the delayed strokes) for the final recognition. An initial result of the algorithm promises the character recognition accuracy of $94.2 \%$.

\section{References}

[1] Plamondon, R., \& Srihari, N. (2000). On-line and off-line handwriting recognition: A comprehensive survey. IEEE Transactions on Pattern Analysis and Machine Intelligence, 22(1), 63-84.

[2] Ghods, V., \& Kabir, E. (2011). Lexicon reduction using delayed strokes for the recognition of online farsi subwords. Proceedings of the 3rd International Conference on Computer Engineering and Technology (ICCET) (pp. 125-129).

[3] Ghods, V., Kabir, E., \& Razzazi, F. (2013). Decision fusion of horizontal and vertical trajectories for recognition of online Farsi subwords. Engineering Applications of Artificial Intelligence, 26(1), 544-550.

[4] Ghods, V., \& Kabir, E. (2013). A study on Farsi handwriting styles for online recognition. Malaysian Journal of Computer Science, 26(1), 44-59.

[5] Ghods, V., \& Sohrabi, M. K. (2014). Online Farsi digit recognition using their upper half structure. Proceedings of the SPIE 9443, Sixth International Conference on Graphic and Image Processing (p. 94430B-6).

[6] Tagougui, N., Kherallah, M., \& Alimi, A. M. (2013). Online Arabic handwriting recognition: A survey. International Journal on Document Analysis and Recognition, 16(3), 209-226. 
[7] Ghods, V., Kabir, E., \& Razzazi, F. (2014). Fusion of HMM classifiers, based on x, y and (x, y) signals, for the recognition of online Farsi handwriting: A Large lexicon approach, Arabian Journal for Science and Engineering, 39(3), 1713-1723.

[8] Halavati, R., \& Shouraki, S. B. (2007). Recognition of Persian online handwriting using elastic fuzzy pattern recognition. International Journal of Pattern Recognition and Artificial Intelligence, 21(3), 491-513.

[9] Ghods, V., \& Kabir, E. (2010). Feature extraction for online Farsi characters. Proceedings of the 12th International Conference on Frontiers in Handwriting Recognition (ICFHR) (pp. 477-482).

[10] Razavi, S. M., \& Kabir, E. (2004). A dataset for online Farsi handwriting. Proceedings of the 6th National Conference on Intelligent Systems (in Farsi) (pp. 218-225).

[11] Liwicki, M., \& Bunke, H. (2009). Feature selection for HMM and BLSTM based handwriting recognition of whiteboard notes. International Journal of Pattern Recognition and Artificial Intelligence, 23(5), 907-923.

[12] Ghods, V., Kabir, E., \& Razzazi, F. (2013). Effect of delayed strokes on the recognition of online Farsi handwriting. Pattern Recognition Letters, 34(5), 486-491.

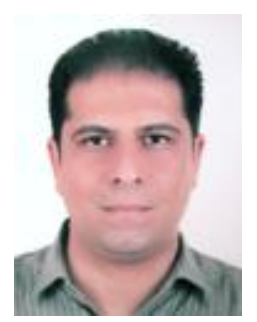

Vahid Ghods was born in Iran in 1981. He received the B.S. degree in electronic engineering from Electrical Engineering Faculty, K N Toosi University of technology (KNTU), Tehran, Iran, in 2002. In 2005, he received the M.S. degree in digital electronic from Electrical Engineering Faculty, Semnan University, Semnan, Iran. He received the Ph.D. degree in electronic from the Electrical Engineering Faculty, Science and Research Branch, Islamic Azad University, Tehran, Iran, in 2012.

He is an assistant professor of Engineering Faculty, Semnan Branch, Islamic Azad University, Semnan, Iran. He has more than 30 of journal and conference publications describing his research area. His research fields are image and video processing, machine vision, speech processing and recognition, artificial intelligence and specially OCR and handwriting recognition.

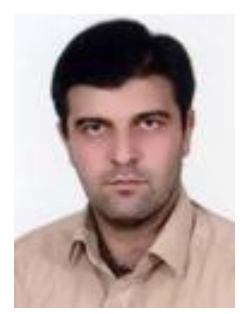

Mohammad Karim Sohrabi was born in Iran in 1980. He received the B.S. degree in software engineering from the Computer Engineering Faculty, Ferdowsi University of Mashhad, Iran, in 2002. In 2005, he received the M.S. degree in software engineering from Computer Faculty, Amirkabir University of Technology (Polytechnic of Tehran), Tehran, Iran. He received the Ph.D. degree in software engineering from the Computer Faculty, Amirkabir University of Technology (Polytechnic of Tehran), Tehran, Iran, in 2012.

He is an assistant professor of Engineering Faculty, Semnan Branch, Islamic Azad University, Semnan, Iran. He has more than 20 of journal and conference publications describing his research area. His research fields are data mining, data warehousing, software engineering, parallel and distributed systems, artificial intelligence and specially frequent pattern mining. 